\title{
Spatial Reasoning with Applications to Mobile Robotics
}

\author{
Lech Polkowski and Pawel Osmialowski \\ Polish-Japanese Institute of Information Technology \\ Poland
}

\section{Introduction to Qualitative Spatial Reasoning}

Qualitative Reasoning aims at studying concepts and calculi on them that arise often at early stages of problem analysis when one is refraining from qualitative or metric details, cf., [14]; as such it has close relations to the design, cf., [10] as well as planning stages, cf., [29] of the model synthesis process. Classical formal approaches to spatial reasoning, i.e., to representing spatial entities (points, surfaces, solids) and their features (dimensionality, shape, connectedness degree) rely on Geometry or Topology, i.e., on formal theories whose models are spaces (universes) constructed as sets of points; contrary to this approach, qualitative reasoning about space often exploits pieces of space (regions, boundaries, walls, membranes) and argues in terms of relations abstracted from a common-sense perception (like connected, discrete from, adjacent, intersecting). In this approach, points appear as ideal objects (e.g., ultrafilters of regions/solids [78]).

Qualitative Spatial Reasoning has a wide variety of applications, among them, to mention only a few, representation of knowledge, cognitive maps and navigation tasks in robotics (e.g. [39], [40], [41], [1], [3], [21], [37], [26]), Geographical Information Systems and spatial databases including Naive Geography (e.g., [24], [25], [33], [22]), high-level Computer Vision (e.g. [84]), studies in semantics of orientational lexemes and in semantics of movement (e.g. [6], [5]). Spatial Reasoning establishes a link between Computer Science and Cognitive Sciences (e.g. [27]) and it has close and deep relationships with philosophical and logical theories of space and time (e.g., [65], [8], [2]). A more complete perspective on Spatial Reasoning and its variety of themes and techniques may be acquired by visiting one of the following sites: [75], [83], [56].

Any formal approach to Spatial Reasoning requires Ontology, cf., [32], [70], [11]. In this Chapter we adopt as formal Ontology the ontological theory of Lesniewski (cf. [49], [50], [69], [47], [36], [18]). This theory is briefly introduced in Section 2.

For expressing relations among entities, mathematics proposes two basic languages: the language of set theory, based on the opposition element-set, where distributive classes of entities are considered as sets consisting of (discrete) atomic entities, and languages of mereology, for discussing entities continuous in their nature, based on the opposition partwhole. Due to this, Spatial Reasoning relies to great extent on mereological theories of part, cf., [4], [5], [6], [12], [15], [30], [31], [28], [71], [72], [55]. 
Mereological ideas have been early applied toward axiomatization of geometry of solids, cf., [45], [78]. Mereological theories dominant nowadays come from ideas proposed independently by Stanislaw Lesniewski and Alfred North Whitehead.

Mereological theory of Lesniewski is based on the notion of a part (proper) and the notion of a (collective) class, cf., [49], [51], [18], [73], [52]. Mereological ideas of Whitehead based on the dual to part notion of an extension [85] were formulated in the Calculus of Individuals [46] and given a formulation in terms of the notion of a connection [12]. Mereology based on connection gave rise to spatial calculi based on topological notions derived therefrom (mereotopology) cf. [16], [14], [20], [23], [5], [6], [15], [30], [31], [28], [71], [55].

Our approach to spatial reasoning is based on the paradigm of rough mereology, see sect. 4 . Rough mereology is based on the predicate of being a part to a degree and thus it is a natural extension of mereology based on part relation.

We demonstrate that in the framework of rough mereology one may define a quasi-Čech topology [19] (a quasi - topology was introduced in the connection model of mereology [12], [5] under additional assumptions of regularity). By a quasi-topology we mean a topology without the null element (being the equivalent of the empty set).

Finally, we apply rough mereology toward inducing geometrical notions. It is well known, cf., e.g., [79], [8] that geometry may be introduced via notions of nearness, betweenness etc. In Section 7, we define these notions by means of a rough mereological notion of distance and we show that in this way a geometry may be defined in the rough mereological universe. This geometry is clearly of approximate character, approaching precise notions in a degree due to uncertainty of knowledge encoded in rough inclusions.

We show applications of the proposed scheme to localization and navigation by a mobile robot equipped with sonar sensors in an environment endowed with a GPS system and we discuss its implementations in the Player/Stage system.

\section{Ontology of spatial objects}

In reasoning with spatial objects, of primary importance is to develop an ontology of spatial objects, taking into account complexity of these objects. We propose a hierarchical ontology obtained by iterative application of the Lesniewski ontological principle. Ontology was intended by Stanislaw Lesniewski as a formulation of general principles of being [50], cf., also [36], [47], [69], [35]. In application-oriented spatial reasoning systems, ontology appears as typology of concepts and their successive taxonomy, cf., e.g., [54] (to quote a small excerpt: edge is frontier, barrier, dam, cliff, shoreline).

The only primitive notion of Ontology of Leśniewski is the copula " is".

We begin with the axiom of Ontology.

The Leśniewski Ontological Axiom is formulated in terms of the conjunctive is whose intuitive semantics denotes the fact that one object (individual) falls under the scope of a collective notion (a collection of individuals).

We assume a set of primitive objects $S$, a set of complex objects $C$, and we formulate a restricted ontological axiom:

$$
X \text { is } Y \text { iff }(X \in S) \wedge(Y \in C) \wedge(\text { for all } Z \in S . Z \text { is } X \Rightarrow Z \text { is } Y \text { ). }
$$

The meaning is: $X$ is an object from $S$ and $X$ belongs in $Y$ extensively (anything in $S$ which is $X$ is also $Y$ ). 
Example 1 Assume a unit grid on the Euclidean d-space $R^{d}$, let $S_{1}$ be the collection of unit cubes resulting from the grid, and $C_{1}$ a non-empty collection of connected unions of finitely many cubes (one may look at this as a collection of obstacles built from single cubes). On the next level, $S_{2}$ can be taken as $C_{1}$, and $C_{2}$ can be defined as a collection of finite unions of members of $C_{1}$ which are pairwise disjoint (making an environment map of obstacles).

Example $2 S$ is a collection of closed discs, and $C$ is a collection of finite unions of (eventually, pairwise disjoint) closed discs.

Extending the idea just put forth, we define a Layered Ontology (LO) as a sequence $\left(\left(S_{i}, C_{i}\right): i=1,2, \ldots\right)$ such that:

LO1. $\left(S_{i}, C_{i}\right)$ are related by the Ontology Axiom for each $i$;

LO2. $S_{i+1}=C_{i}$ for each $i$;

LOS. $C_{i}=F_{i}\left(S_{i}\right)$ for each $i$, where $F_{i}$ is an operator (in examples above it was taken as the union).

Ontological theories play an important role in Approximate Reasoning [11], [32], [70] witnessed with particular clearness in Spatial Reasoning [54], [22] where Ontology plays a basic role as it sets spatial concepts and their taxonomy.

\section{Mereology}

Yet another source of ideas and points of reference for rough mereology are mereological theories of concepts/sets. We refer here to two mainstream theories of mereology, viz., mereology due to Lesniewski [49], [51], [52], [73], [74], [18], [79], [13], [48] and mereology based on the notion of connection [12], [46], [85], [15], [16], [55], [4], [6].

Of the two theories, mereology based on connection offers a richer variety of mereotopological functors; mereology based on the notion of part offers a formalism of which the formalism of rough mereology is a direct extension and generalization: the latter was proposed [58], [59], [61], [62] to contain mereology as the theory of the predicate $\mu_{1}$.

\subsection{Mereology based on parts}

We denote with the symbol $p t$ the relation of part on a collection of objects, subject to conditions,

PI. $X$ is $p t Y \Rightarrow X$ is $X \wedge Y$ is $Y$ (the relation $p t$ is defined for individual entities only);

P2. $X$ is $p t Y \wedge Y$ is $p t Z \Rightarrow X$ is $p t Z$ ( $p t$ is transitive);

P3. $\neg(X$ is $p t X)(p t$ is non-reflexive).

On the basis of the notion of part, we define the notion of an element (an improper part; called originally in [49], an ingredient) as a relation $\mathrm{el}$ :

$$
X \text { is } e l Y \Leftrightarrow X \text { is } p t Y \vee X=Y \text {. }
$$

The remaining axioms of mereology are related to the class functor which converts distributive classes (general names) into individual entities. The class operator $\mathrm{Cl}$ is a principal tool in applications, cf., [58], [59], [61], [62], [68].

We may now introduce the notion of a (collective) class via the class functor $\mathrm{Cl}$.

\subsubsection{The class operator}

An individual $X$ is the class of a non-vacuous collection $M$ of objects, in symbols, $X$ is $\mathrm{ClM}$, if 
Cl1. If $Y \in \mathcal{M}$ then $Y$ el $X$;

C12. $\forall Z$. $(Z$ is el $X \Rightarrow \exists U, W$. $(U$ is $Y \wedge W$ is $e l l \wedge W$ is $e l Z)$.

Let us disentangle the meaning of this definition. First, we may realize that the class operator $\mathrm{Cl}$ is intended as the operator converting names (general sets of entities) into individual entities i.e. collective classes; its role may be fully compared to the role of the union of sets operator in the classical set theory. The analogy is indeed not only functional but also formal.

By $\mathrm{Cl1}$., the class contains any member of the collection; by $\mathrm{Cl2}$., the class contains all objects $Z$ with the property: each element of $Z$ has an element in common with an object in the collection.

Thus, the class functor pastes together individuals in $Y$ by means of their common elements. A basic tool in reasoning by means of mereology, see [51], is the following inference rule, IR. For $X, Y$ : if for each $Z$ (from $Z$ is el $X$ it follows that there is $T$ such that $T$ is $e l Z \wedge T$ is $e l$ $Y)$ then $X$ is el $Y$.

\subsection{Mereology based on connection}

This approach [85], [46], [12] is based on the functor of being connected; for the uniformity of exposition sake, we will formulate all essentials of this theory in the ontology language applied above.

The requirements for a functor Con of connection are as follows,

Conl. $X$ is $\operatorname{Con} Y=>X$ is $X \wedge Y$ is $Y$ (asserting that Con is defined on individuals);

Con2. $X$ is Con $X$ (asserting refiexivity of Con);

Con3. $X$ is $\operatorname{Con} Y \Leftrightarrow Y$ is Con $X$ (asserting that Con is symmetric);

Con4. For all $Z$ ( $Z$ is $\operatorname{Con} X \Leftrightarrow Z$ is $\operatorname{Con} Y \Rightarrow X=Y$ ) (asserting extensionality of Con).

From the functor $C o n$, other functors are derived,

DCon. $X$ is $D \operatorname{Con} Y \Leftrightarrow \operatorname{non}(X$ is $\operatorname{Con} Y)$ ( $X$ is disconnected from $Y)$;

$E l_{\text {c. }} X$ is $e l_{C} Y \Leftrightarrow$ for all $Z(Z$ is $\operatorname{Con} X) \Rightarrow Z$ is $\left.\operatorname{Con} Y\right)(X$ is a connection element of $Y)$;

$\mathrm{P}$. $X$ is $\mathrm{P} Y \Leftrightarrow X$ is $e l_{C} Y \wedge n o n\left(Y\right.$ is $\left.e l_{C} X\right)$ ( $X$ is a part of $\left.Y\right)$;

Ov. $X$ is $O v Y \Leftrightarrow$ exists $Z\left(Z\right.$ is $\left.e l_{C} X\right) \wedge Z$ is $\left.e l_{C} Y\right)(X, Y$ overlap $)$;

ECon. $X$ is .ECon $Y \Leftrightarrow X$ is $\operatorname{Con} Y \wedge$ non ( $X$ is $O v Y)$ ( $X$ is externally connected to $Y$ );

TP. $X$ is TP $Y \Leftrightarrow X$ is $P Y \wedge$ exists $Z$ ( $Z$ is .ECon $X \wedge Z$ is .ECon $Y$ ) ( $X$ is a tangential part of $Y)$;

NTP. $X$ is NTP $Y \Leftrightarrow X$ is $P Y \wedge$ non $(X$ is TP $Y)(X$ is a non-tangential part of $Y$ ).

Connection allows for a variety of functors of topological characters (one may define a quasi-topological interior by means of NTP, cf., eg., [5], [6], [12], [55]).

\section{Rough mereology}

Rough mereology, see [59], [60], [61], [62] begins with the notion of a rough inclusion which is a parameterized relation $\mu_{r}$ such that for any pair of individual entities $X, Y$ the formula $Y$ is $\mu_{r} X$ means that $\mathrm{Y}$ is a part of $X$ to a degree $r$ where $r \in[0,1]$.

The following is the list of basic postulates for rough inclusions; $e l$ is the element relation of a chosen mereology system.

RM1. $X$ is $\mu_{1} \mathrm{Y} \Leftrightarrow X$ is $e l(Y)$ (a part in degree 1 is equivalent to an element);

RM2. $X$ is $\mu_{1} Y \Rightarrow$ for all $Z$ ( $Z$ is $\mu_{r} X=>Z$ is $\mu_{r} Y$ ) (monotonicity of $\mu$ );

RM3. $X$ is $\mu_{r} Y \wedge \mathrm{s} \leq r \Rightarrow X$ is $\mu_{s} Y$ (assuring the meaning "a part in degree at least $r^{\prime \prime}$ ). 


\subsection{Rough inclusions on collections of objects}

Assume that we are given two individuals $X, Y$ being classes of (finite) names:

$\mathrm{X}=\mathrm{Cl}\left(\mathrm{X}^{\prime}\right), \mathrm{Y}=\mathrm{Cl}\left(\mathrm{Y}^{\prime}\right)$ and that we have defined values of $\mu$ for pairs $T, \mathrm{Z}$ of individuals where Tis $X^{\prime}, Z$ is $Y^{\prime}$.

We extend $\mu$ to a measure $\mu^{*}$ on $X, Y$ by letting:

$$
r=\min _{Z \in Y^{\prime}}\left\{\max _{T \in X^{\prime}} \max \left\{s: Z \text { is } \mu_{s}(T)\right\}\right\} \quad \text { and } \quad Y \text { is } \mu_{r}^{*}(X) .
$$

It may be proved straightforwardly that the measure $\mu^{*}$ satisfies (RM1) - (RM4).

\subsection{Transitive rough inclusions}

We introduce now [58], a modification to our functors $\mu_{r}$; it is based on an application of residuated implication [38] and a measure of containment defined within the fuzzy set theory (the necessity measure) [34] , [7] . Combining the two ideas, we achieve a formula for $\mu_{r}$ which allows for a transitivity rule; this rule will in turn allow to introduce into our universe rough mereological topologies. We therefore recall the notion of a $t$-norm $T$ as a function of two arguments $T:[0,1]^{2} \rightarrow[0,1]$ which satisfies the following requirements:

2. $\top(x, y)=\top(y, x)$;

3. $\top(x, \top(y, z))=\top(\top(x, y), z)$;

4. $\top(x, 1)=x$;

5. $\quad x^{\prime} \geq x \wedge y^{\prime} \geq y \Rightarrow \top\left(x^{\prime}, y^{\prime}\right) \geq \top(x, y)$.

We also invoke a notion of fuzzy containment $C_{r}$ based on necessity, cf., [34]; it relies on a many-valued implication $\Upsilon$ i.e. on a function $\Upsilon:[0,1]^{2} \Rightarrow[0,1]$ according to the formula:

$$
X \subset_{r} Y \Longleftrightarrow \forall Z .\left(\Upsilon\left(\mu_{X}(Z), \mu_{Y}(Z)\right) \geq r\right),
$$

where $\mu_{\mathrm{A}}$ is the fuzzy membership function [38] of the fuzzy set $A$.

We replace $\Upsilon$ with a specific implication, viz., the residuated implication $\vec{T}$ induced by $\top$ and defined by the formula: $\vec{\top}(r, s) \geq t \Longleftrightarrow \top(t, r) \leq s$.

We define a predicate $\mu_{\top, r}$ where $r \in[0,1]$, according to the formula:

$$
X \text { is } \mu_{\top, r} Y \Longleftrightarrow \text { for all } Z \text { (exist } t, w\left(Z \text { is } \mu_{t} X \wedge Z \text { is } \mu_{w} Y \wedge \vec{\top}(t, w) \geq r\right. \text {.) }
$$

As proved in a different context in [58], $\mu_{\top, r}$ satisfies (RM1-RM4).

The rough inclusion $\mu_{\top, r}$ does satisfy a deduction rule of the form, DR. If $X$ is $\mu_{\top, r} Y$ and $Y$ is $\mu_{\top, s} Z$ then $X$ is $\mu_{\top, T(r, s)} Z$.

We now propose to synthesize basic topological and geometric constructs applied in Qualitative Spatial Reasoning based on connection, e.g., [6], [5], by means of rough mereology.

\section{Mereotopology}

As mentioned few lines above, topological structures may be defined within the connection framework via the notion of a non - tangential part. Interior entities are formed then by means of some fusion operators, see, e.g. [5], [55]. The functor of connection allows also for some calculi of topological character based directly on regions, e.g., RCC - calculus, see, 
[31]. For a different approach where connection may be derived from the axiomatized notion of a boundary, see, [72].

These topological structures provide a mereotopological environment in which it is possible to carry out spatial reasoning. We now demonstrate that in rough mereological framework one defines in a natural way Cech topologies.

We would like to recall that a topology on a given domain $U$ may be introduced by means of a closure operator $c l$ satisfying the Kuratowski axioms [43]:

(cl1) $c l \varnothing=\varnothing$;

(cl2) $c l c l X=c l X$;

(cl3) $X \subseteq c l X ;$

(cl4) $c l(X \cup Y)=c l X \cup c l Y$.

The dual operator int of interior is then defined by means of the formula: int $X=U-\operatorname{cl}(U-$ $X)$ and it has dual properties: $\operatorname{int} \varnothing=\varnothing, \operatorname{intint} X=\operatorname{int} X, \operatorname{int} X \subseteq X, \operatorname{int}(X \cap Y)=\operatorname{int} X \cap$ int $Y$.

The Čech topology [19] is a weaker structure as it is required here only that the closure operator satisfies the following:

(Čcl1) $c l \varnothing=\varnothing$;

(Čcl2) $X \subseteq c l X ;$

(Čcl3) $X \subseteq Y \Rightarrow d X \subseteq d Y$.

so the associated Čech interior operator int should only satisfy the following: int $\varnothing=\varnothing$; int $X \subseteq X ; X \subseteq Y \Rightarrow$ int $X \subseteq$ int $Y$.

Čech topologies arise naturally in problems when one considers coverings induced by similarity relations [53].

In order to define Čech topologies, we first define the class $\mathrm{Cl}_{r} X$ for any object $X$ and $r<1$, as the class of objects having the property $M_{r} X$ of being a part of $X$ to a degree $r: C l_{r} X=C l$ $M_{r} X$.

By means of the rule (DR), sect.4.2, one can establish the properties,

Mon. For $s \leq r, \mathrm{Cl}_{r} \mathrm{X}$ is $\mathrm{el} \mathrm{Cl}_{s} \mathrm{X}$.

Her. Xis el $Y \Rightarrow \mathrm{Cl}_{r}$ Xis $e l \mathrm{Cl}_{r} Y$.

Following this, we define a new functor int as the class of the property $I(X)$, int $X=C l I(X)$, where $\mathrm{Zis} I(X) \Leftrightarrow$ exists $s<1\left(C l_{s} Z\right.$ is $\left.e l X\right)$.

We have the following properties of int,

I1. $\operatorname{int}(X)$ is el $X$;

I2. $X$ is el $Y \Rightarrow \operatorname{int}(X)$ is $\operatorname{el} \operatorname{int}(Y)$.

Properties (I1)-(I2) witness this quasi-topology is a quasi-Čech topology. We denote it by the symbol $\tau_{\mu}$.

We now study the case of mereotopology under functors $\mu_{\mathrm{T}, r}$ in this case, the quasi- Čech topology $\tau_{\mu}$ turns out to be a quasi-topology.

\subsection{Mereotopology in the case of $\mu_{\mathrm{T}}$}

We begin with an application of deduction rule (DR). We denote by the symbol $C l_{\top . r} X$ the set $C l_{r} X$ in case of the rough inclusion $\mu$. We assume that $\top(r, s)<1$ when $r s<1$. We have a new direct characterization of $C l_{\top, r} X: Z$ is $e l C l_{\top . r} X \Leftrightarrow Z$ is $\mu_{\top, r} X$.

This characterization implies that $C l_{\top . r} X$ may be regarded as "an open ball of radius $r$ centered at $X^{\prime \prime}$. 
We assume now, additionally, that the t-norm $\top$ has the property that: for every $r<1$ there exists $s<1$ such that $\top(r, s)>r$. With this assumption, we have the following property, INC. For Zis $e l C l_{\top . r} X$, if $s_{0}=\arg \_\min \{s: \top(r, s) \geq r\}$ then

$$
C l_{\top, s_{0}} \mathrm{Z} \text { is } e l\left(C l_{\top . r} \mathrm{X}\right) \text {. }
$$

We define a functor of two nominal individual variables $A N D$, AND. Zis el $A N D(X, Y) \Leftrightarrow$ $O v(X, Y) \wedge$ Zis el $X \wedge$ Zis el $Y$.

The rough mereotopology $\tau_{\mu_{\top}}$ has the properties:

INTER. $A N D(\operatorname{int}(X), \operatorname{int}(Y))=\operatorname{int}(A N D(X, Y))$ holds whenever

$\operatorname{AND}(\operatorname{int}(X), \operatorname{int}(Y))$ is non-empty.

IDEM. $\operatorname{int}(\operatorname{int}(X))=\operatorname{int}(X)$.

It follows by INTER and IDEM that the rough mereological topology induced by the rough inclusion $\mu_{\mathrm{T}, r}$ is a quasi-topology.

\section{Connections from Rough Inclusions}

In sect. 3.2, we presented basic notions related to mereological theories based on the notion of a connection. We recall that a connection is a functor which satisfies axioms (Conl)-(Con4) of sect. 3.2.

In this section we will investigate some methods for inducing connections from rough inclusions. Clearly, the presence of topology induced in the preceding section allows for a few approaches to this problem. We begin with a notion of a connection in a strong sense.

\subsection{Strong connection}

We define a name-forming functor $C$ on $n_{T}$ on individual entities as follows, $\operatorname{CON}(\mathrm{T})$. $\mathrm{Xis} C \operatorname{on}_{T} Y \Leftrightarrow \operatorname{non}\left(\right.$ exist $r, s<1$.ext $\left(\mathrm{Cl}_{r} \mathrm{X}, \mathrm{Cl}_{s} Y\right)$ ).

Thus, $X$ and $Y$ are connected in the strong sense whenever they cannot be separated by means of their open neighborhoods.

We check whether $C$ on $_{T}$ thus defined does satisfy (Conl)-(Con4). It may be clear that (Conl), (Con2), (Con3) hold irrespective of properties of $\mu$. The status of (Con4) will clearly depend on our assumed functor $\mu$. In case non $(X=Y)$, we have, e.g., $Z$ is el $X)$, ext $(Z, Y)$ with some $Z$. Clearly, $Z$ is $C o n_{T} X$; to prove that $n o n\left(Z\right.$ is $\left.C o n_{T} Y\right)$, we need some assumptions about the form of $\mu$.

We add a new property of $\mu$,

RM4. If it is not true that $X$ is $O v \mathrm{Y}$, then there exists $s<1$ such that (if $X$ is $\mu_{r} Y$ then $r<s$ ). Assume (RM4) and consider $\mu_{\top}^{s}$ with a t-norm $T$ which would satisfy the following: given $s$ $<1$, there exist $\alpha, \beta<1$ with the property that $T(\alpha, \beta)>s$. Then, $C$ on $n_{T}$ induced via $\mu_{T}^{s}$ would satisfy (Con4).

In connection framework, the notion of an element is derived from the functor $C$ of connection; the resulting functor of an element is denoted here by the symbol $e l_{C}$ We will find relationships between the original functor $\mathrm{el}$ of an element and the functor $\mathrm{el}_{\mathrm{C}}$. To this end, we have

ELEM1. For any functor of the form $\mu_{\top}$ : $X$ is $e l Y=>X$ is $e l_{C_{T}} Y$.

ELEM2. For any functor of the form $\mu_{\top}^{s}: X$ is $e l_{C_{T}} Y=>$ Xis el $Y$.

A corollary follows,

For any functor of the form $\mu_{\top}^{s}: e l_{C_{T}}$ and $e l$ are equivalent. 
We may therefore create in the framework of rough mereology an alternative scheme of calculus of individuals based on the connection $C_{T}$ inducing the same notion of an element as the original mereological one.

\section{Mereogeometry}

Predicates $\mu_{r}$ may be regarded as weak metrics also in the context of geometry. From this point of view, we may apply $\mu$, in order to define basic notions of rough mereological geometry.

In the language of this geometry, we may approximately describe and approach geometry of objects described by data tables; a usage for this geometry may be found, e.g., in navigation and control tasks of mobile robotics [1], [3], [21], [37], [40], [41].

It is well-known (see, [80], [8]) that the geometry of Euclidean spaces may be based on some postulates about the basic notions of a point and the ternary equi-distance functor. In [80], postulates for Euclidean geometry over a real-closed field were given based on the functor of betweenness and the quaternary equi-distance functor. Similarly, in [8], a set of postulates aimed at rendering general geometric features of geometry of finitedimensional spaces over reals has been discussed, the primitive notion there being that of nearness.

Geometrical notions have been applied in, e. g., studies of semantics of spatial prepositions [6] and in inferences via cardinal directions [42].

\subsection{Rough mereological distance, betweenness}

We first introduce a notion of distance $\kappa_{r}$ in our rough mereological universe by letting

$$
\kappa_{r}(X, Y) \Longleftrightarrow r=\min \left\{\max u, \max w: X \text { is } \mu_{u} Y \wedge Y \text { is } \mu_{w} X\right\} .
$$

We now introduce the notion of betweenness as a functor $T(X, Y)$ of two individual names; the statement $Z$ is $T(X, Y)$ reads as ' $Z$ is between $X$ and $Y$ :

$$
Z \text { is } T(X, Y) \Longleftrightarrow \text { for all W } \kappa_{r}(Z, W) \wedge \kappa_{s}(X, W) \wedge \kappa_{t}(Y, W) \Longrightarrow s \leq r \leq t \vee t \leq r \leq s .
$$

Thus, $Z$ is $T(X, Y)$ holds when the rough mereological distance $\kappa$ between $Z$ and any $W$ is in the non-oriented interval (i.e. between) [distance of $X$ to $W$, distance of $Y$ to $W$ ] for any $W$. One checks that $T$ satisfies the axioms of Tarski [80] for betweenness.

Proposition 1 The following properties hold:

1. $Z$ is $T(X, X)=>Z=X$ (identity);

2. $Y$ is $T(X, U) \wedge Z$ is $T(Y, U) \Rightarrow Y$ is $T(X, Z)$ (transitivity);

3. $Y$ is $T(X, Z) \wedge Y$ is $T(X, U) \wedge X \neq Y \Rightarrow Z$ is $T(X, U) \vee U$ is $T(X, Z)$ (connectivity).

Proof 1 By means of $\kappa$, the properties of betweenness in our context are translated into properties of betweenness in the real line which hold by the Tarski theorem [80], Theorem 1.

\subsection{Nearness}

We may also apply $\kappa$ to define in our context the functor $N$ of nearness proposed in van Benthem [8]:

$$
Z \text { is } N(X, Y) \Longleftrightarrow\left(\kappa_{r}(Z, X) \wedge \kappa_{s}(X, Y) \Longrightarrow s<r\right) \text {. }
$$


Here, nearness means that $Z$ is closer to $X$ than to $Y$ (recall that rough mereological distance is defined in an opposite way: the smaller $r$, the greater distance).

Then the following hold, i.e., $N$ does satisfy all axioms for nearness in [8],

Proposition 2 1. Zis $N(X, Y) \wedge Y$ is $N(X, W) \Rightarrow Z$ is $N(X, W)$ (transitivity);

2. Zis $\mathrm{N}(\mathrm{X}, \mathrm{Y}) \wedge \mathrm{X}$ is $\mathrm{N}(\mathrm{Y}, \mathrm{Z}) \Rightarrow \mathrm{Xis} N(\mathrm{Z}, \mathrm{Y})$ (triangle inequality);

3. $\operatorname{non}(Z i s N(X, Z)$ ) (irreflexivity);

4. $Z=X \vee Z$ is $N(Z, X)$ (selfishness);

5. Zis $N(X, Y) \Rightarrow Z$ is $N(X, W) \vee$ Wis $N(X, Y)$ (connectedness).

Proof 2 (4) follows by (RM1); (3) is obvious. In proofs of the remaining properties, we introduce a symbol $\mu(X, Y)$ as a value of $r$ for which $\kappa_{r}(X, Y)$. Then, for $(l)$, assume that $Z$ is $N(X, Y), Y$ is $N(X, W)$ i.e. $\mu(Z, X)>\mu(X, Y), \mu(X, Y)>\mu(X, W)$ hence $\mu(Z, X)>\mu(X, W)$ i.e. $Z$ is $N(X, W)$. In case (2), Zis $N(X, Y)$, Xis $N(Y, Z)$ mean $\mu(Z, X)>\mu(X, Y), \mu(X, Y)>\mu(Y, Z)$ so $\mu(Z, X)>\mu(Y, Z)$ i.e. $X i s N(Z, Y)$. Concerning $(v)$, Zis $N(X, Y)$ implies that $\mu(Z, X)>\mu(X, Y)$ hence either $\mu(Z, X)>\mu(X$, $W)$ meaning $Z$ is $N(X, W)$ or $\mu(X, W)>\mu(X, Y)$ implying Wis $N(X, Y)$.

We now may introduce the notion of equi-distance as a functor $E q(X, Y)$ defined as follows:

$$
Z \text { is } E q(X, Y) \Longleftrightarrow(\operatorname{non}(X \text { is } N(Z, Y)) \wedge \operatorname{non}(Y \text { is } N(Z, X))) \text {. }
$$

It follows that

Proposition $3 \mathrm{Zis} E q(X, Y) \Leftrightarrow$ for all $\left(\kappa_{r}(X, Z) \Leftrightarrow \kappa_{r}(Y, Z)\right)$.

We may also define a functor of equi-distance following Tarski [80]:

$$
\left.D(X, Y, Z, W) \Longleftrightarrow \text { (for all } \mathrm{r} \kappa_{r}(X, Y) \Longleftrightarrow \kappa_{r}(Z, W)\right) \text {. }
$$

These functors do clearly satisfy the following (see, [8], [80])

Proposition 4 1. Zis $E q(X, Y) \wedge X i s E q(Y, Z) \Rightarrow Y$ is $E q(Z, X)$ (triangle equality);

2. Zis $T(X, Y) \wedge$ Wis $E q(X, Y)=\wedge>D(Z, W, X, W)$ (circle property);

3. $D(X, Y, Y, X)$ (reflexivity);

4. $D(X, Y, Z, Z) \Rightarrow X=Y$ (identity);

5. $D(X, Y, Z, U) \wedge D(X, Y, V, W) \Rightarrow D(Z, U, V, W)$ (transitwity).

One may also follow van Benthem's proposal for a betweenness functor defined via the nearness functor as follows:

$$
\left.Z \text { is } T_{B}(X, Y) \Longleftrightarrow \text { [for all } W(Z \text { is } W \vee Z \text { is } N(X, W) \vee Z \text { is } N(Y, W))\right] \text {. }
$$

One checks in a straightforward way that

Proposition 5 The functor $T_{B}$ of betweenness defined according to the above does satisfy the Tarski axioms.

\subsection{Points}

The notion of a point may be introduced in a few ways; e.g. following Tarski [78], one may introduce points as classes of names forming ultrafilters under the ordering induced by the functor of being an element $e l$. Another way, suitable in practical cases, where the universe, or more generally, each ultrafilter $F$ as above is finite, i.e., principal (meaning that there exists an object $X$ such that $F$ consists of those $Y$ "'s for which Xis el( $Y$ ) holds) is to define 
points as atoms of our universe under the functor of being an element i.e. we define a constant name AT as follows:

$$
X \text { is } A T \Longleftrightarrow n o n(\text { exists } Y(Y \text { is } e l(X) \wedge \operatorname{non}(X \text { is }=Y) \text {. }
$$

We will refer to such points as to atomic points. We adopt here this notion of a point. Clearly, restricting ourselves to atomic points, we preserve all properties of functors of betweenness, nearness and equi-distance proved above to be valid in the universe $V$.

\subsection{Mereogeometry: Examples}

We will adopt the notion of betweenness $T_{B}$ based on the nearness functor.

We give some examples of specific contexts in which this functor can be realized.

Example 3 With reference to Example 1, we adopt as objects topologically connected unions of finitely many cubes in the unit grid on the space $R^{d}$ (topological connectedness will be defined recursively: (1). a single cube is connected; (2) given a connected union $C$ and a cube $c$, the union $\mathrm{C} U_{c}$ is connected if $c$ is adjacent by the edge or a vertex to a cube in $\left.C\right)$. We adopt as the rough inclusion $H$ the function $\mu(C, D, r)$ iff $\frac{|C \cap D|}{|C|} \geq r$, where $|C \cap D|$ is the number of cubes common to $C$ and $D$ and $|C|$ is the number of cubes in $C$. The mereological distance between $C$ and $D$ is then: $\kappa(C, D)=\min \{\max \{r: \mu(C, D, r)\}, \max \{s: \mu(D, C, s)\}\}$.

Then one checks that: the connected union $E$ is between (in the sense of $T_{B}$ ) disjoint cubes $c, d$ whenever $E$ contains $c$ and $d$ and $E$ consists of a minimal number of cubes for $E$ being connected.

One can interpret this result as follows: when c,d are obstacles (e.g., of the size of a mobile robot) then $\mathrm{Z}$ minus $\mathrm{c}$, $\mathrm{d}$ gives the space between these obstacles, free for the robot to pass.

Example 4 In the same frame and with same $\mu$, consider unions of grid cubes (not necessarily topologically connected). Then for disjoint cubes $c, d$ : a, union $Z$ of grid cubes is between $c, d$ (in the sense of) $T_{B}$ whenever $Z$ is either $c$, or $d$, or $c \bigcup d$.

One can interpret this as: when unit grid is of the size of a mobile robot, $Z$ between $c$ and $d$, can be interpreted as beacons (landmarks) between which a robot may pass safely.

Example 5 We consider now obstacles modeled as squares on $R^{2}$ of equal size given by edge length $r$, but not necessarily conforming to any grid, i.e., a square can be centered at any point; we change the rough inclusion $\mu$, to the following: $\mu(C, D, r)$ iff $\frac{\|C \cap D\|}{\|C\|} \geq r$, where $\|C\|$ is the area of $C$. In this setting, consider disjoint squares $C, D$ whose centers are on the line e.g., $x=0$.

Then one checks: any square centered on $\mathrm{x}=0$ with the center on the segment joining centers of $C$ and $D$ of edge length $r$ is between $C$, and $D$, in the sense of $T_{B}$.

One can interpret this as follows: a robot of size of the square of edge length $r$, should interpret the space formed by the rectangular space between $C$ and $D$ minus $C, D$ as the eventual free space for bypassing either $C$ or $D$.

Example 6 In the setting of Example 5, we replace squares with discs of equal radius $r$ placed arbitrarily in the space $R^{2}$, with $\mu$, defined as therein by means of the area. Then conclusion holds with disks as with squares. 
Example 7 The equi-distance functor Eq may be used to define spheres; for instance, admitting as $Z$ the square $[0,1] \times[0,1]$, (so the edge length is 1 ) we have the sphere $S(Z ; 1 / 2)$ to contain squares: $[0,1]$ $x[1 / 2,3 / 2],[0,1] \times[-1 / 2,1 / 2],[1 / 2,3 / 2] \times[0,1],[-1 / 2,1 / 2] \times[0,1]\}$.

A line segment may be defined via the auxiliary notion of a pattern; we introduce this notion as a functor Pt. We let

$$
P t(X, Y, Z) \Longleftrightarrow Z \text { is } T_{B}(X, Y) \vee X \text { is } T_{B}(Z, Y) \vee Y \text { is } T_{B}(X, Z) .
$$

We will say that a finite sequence $X_{1}, X_{2}, \ldots, X_{n}$ of objects belong in a line segment whenever $\operatorname{Pt}\left(X_{i}, X_{i+1}, X_{i+2}\right)$ for $i=1, \ldots, n-2$; formally, we introduce the functor Line of finite arity defined via

$$
\operatorname{Line}\left(X_{1}, X_{2}, \ldots, X_{n}\right) \Longleftrightarrow \forall i<n-1 . P t\left(X_{i}, X_{i+1}, X_{i+2}\right) .
$$

Example 8 With reference to Example 5 , consider a sequence of squares $C_{1}, \ldots, C_{k}$ all centered on $x=0$, and of edge length $r$. Then, Line $\left(C_{1}, \ldots, C k\right)$ holds. One can interpret this as a robot moving along a straight line when its consecutive positions are $C_{1}, \ldots, C k$.

The notion of orthogonality may be introduced in a well-known way; we introduce a functor Ortho: for two line segments $A, B$, with an object $Z$ common to sequences $A$ and $B$, we let

$$
\begin{gathered}
\operatorname{Ortho}(A, B) \Longleftrightarrow \exists X, Y, U, W . X, Y \text { is in } A \wedge U, W \text { is in } B \wedge \operatorname{non}(X \text { is } Y) \\
\qquad \operatorname{non}(U \text { is } W) \wedge U \text { is } E q(X, Y) \wedge W \text { is } E q(X, Y),
\end{gathered}
$$

Example 9 With reference to Example 8, consider lines A: the sequence $[0,1] \times[0,1],\{[i / 2, i+1 / 2]$ $\mathrm{x}[0,1]: i=+/-1,+/-2, \ldots\} ; B$ : the sequence $[0,1] \times[0,1],\{[0,1] \times[i / 2, i+1 / 2]: i=+/-!,+/-2, \ldots\}$. Then $\operatorname{Ortho}(A, B)$ holds as witnessed, e.g., by $Z=[0,1] \times[0,1], X=[-1 / 2,1 / 2] \times[0,1], Y=$ $[1 / 2,3 / 2] \times[0,1], U=[0,1] \times[-1 / 2,1 / 2], W=[0,1] \times[1 / 2,3 / 2]$.

Example 10 Finally, we consider a more general context in which objects are rectangles positioned regularly, i.e., having edges parallel to axes in $R^{2}$. The measure $\mu$ is the one in Example 5. In this setting, given two disjoint rectangles $C, D$, the only object between $C$ and $D$ is the extent of $C, D$, $\operatorname{ext}(C, D)$, i.e., the rectangle which is the minimal rectangle containing the union $C \cup D$. To see this, one can consider two identical squares $C, D$ as in Example 5 and solve the problem analytically by showing that there is no other rectangle nearer to $C$ and $D$ (this requires solving a set of linear inequalities); then, the general case follows by observing that linear shrinking or stretching of an edge does not change the area relations.

Rectangles $C, D$ and their extent ext $(C, D)$ form then a line segment.

\section{A.Szmigielski's model for localization and navigation}

In his PhD disssertation [77], A. Szmigielski has proposed an approach to localization and navigation of a mobile robot using the mereological geometry. The robot was a Pioneer $P 2 D X$ endowed with sonar emitter in the environment of sonar sensors.

The control of a robot utilized the parameters,

- length of route segment $d$,

- rotation angle $\alpha$, 
where the robot first traveled the distance $d$ then rotated by $\alpha$. Low level control means a sequence of pairs $(d, \alpha)$. Distance to a nearest obstacle was determined by robot sonars.

The system of sonar sensors can be exploited towards localization of a robot in the global reference frame. The system consists of a sonar emitter positioned on the robot and up to 16 receivers in the environment of the robot. Simultaneous measurement of times of flight between the emitter and receivers gives distances from the robot to receivers. At the same time, robot sonars detect the nearest obstacle.

As the result, two regions are determined in 2D space of the robot plane: the disk centered at the receiver with the radius equal to the distance emitter -receiver (the receiver region), and the disk centered at the robot with the radius equal to the distance to the nearest obstacle (the collision-free region). The robot position is on the boundary of the receiver region.

A relation between the two regions is expressed by the mereodistance $\kappa$.

Whereas the Euclidean distance is context - free, the mereological distance depends on the environment (which bears on radii of regions).

The goal - reaching a desired receiver, can be formulated as the requirement that radii of the receiver and the collision free regions are equal and the distance $\kappa$ between them is maximal $=\frac{2}{3}-\frac{\sqrt{3}}{2 \pi}$.

The stop criterion can be formulated then as the requirement that radii of two regions be equal; then, the receiver is found on the boundary of the collision-free region.

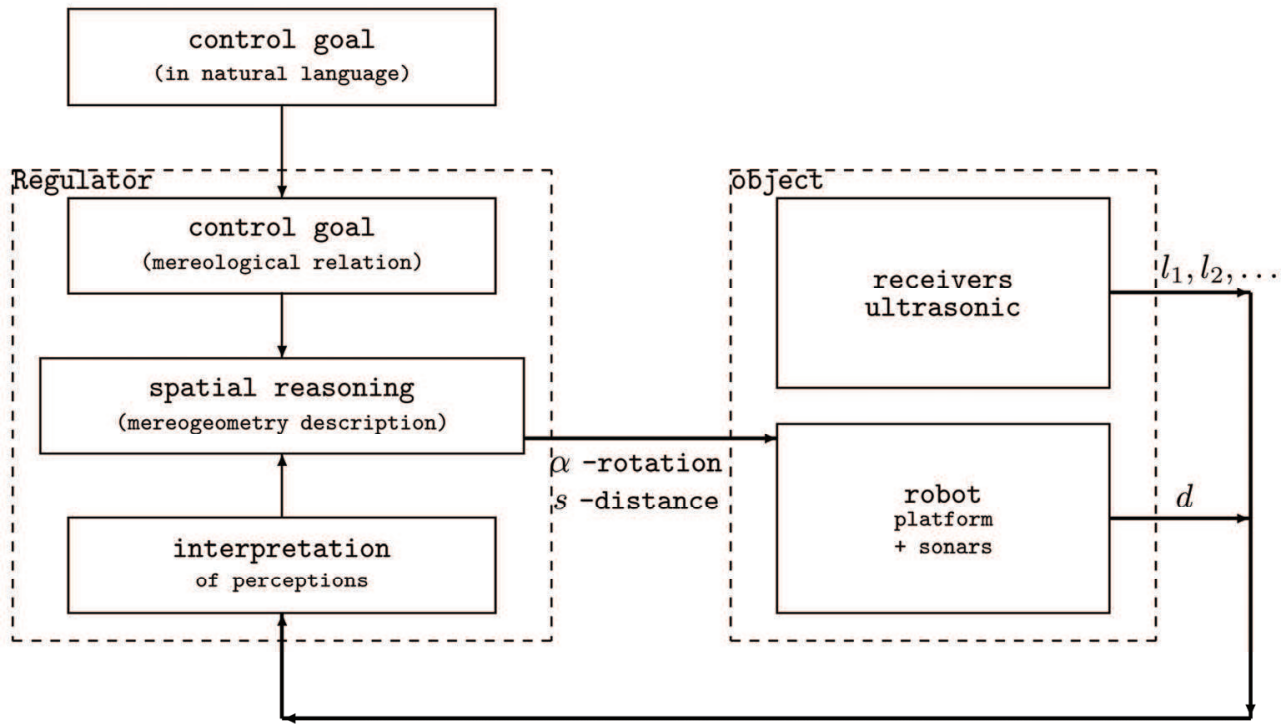

Figure 1. Spatial reasoning in closed loop

Control in dynamic setting is performed in the closed loop: perception - decision - action

- perception. The inference engine gives two parameters:

$s=$ the distance to goal,

$\alpha=$ the rotation angle of the robot. The input parameters are:

$d=$ the radius of the collision - free region, 
$l_{1}, l_{2}, \ldots, l_{k}=$ radii of receiver regions

The block diagram of control is shown in Fig. 1.

Fig. 2 shows the result of the experiment of reaching the goal in an environment with obstacles; trajectory is shown for the robot starting at the orientation of 180 degrees with respect to obstacle.

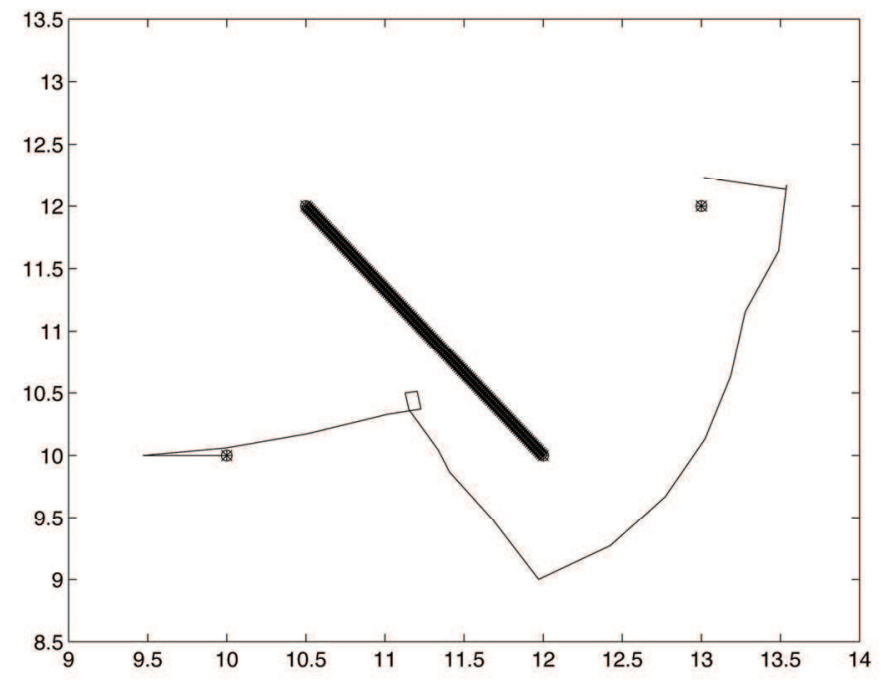

Figure 2. Robot reaches the goal

Further results can be found in [77] and [63].

\section{Implementation in Player/Stage software system}

Player/Stage is an Open-Source software system designed for many UNIX-compatible platforms, widely used in robotics laboratories [57]. Main two parts are Player - message passing server (with bunch of drivers for many robotics devices, extendable by plugins) and Stage - a plug-in for Players bunch of drivers which simulates existence of real robotics devices that operate in simulated 2D world. Player/Stage offers client-server architecture. Many clients can connect to one Player server, where clients are programs (robot controllers) written by a roboticist who can use Player client-side API. Player itself uses drivers to communicate with devices, in this activity it does not make distinction between real and simulated hardware. It gives roboticist means for testing programmed robot controller in both real and simulated world.

Among all Player drivers that communicate with devices (real or simulated), there are drivers not intended for controlling hardware, instead those drivers offer many facilities for sensor data manipulation, for example, camera image compression, retro-reflective detection of cylindrical markers in laser scans, path planning. One of the new features added to Player version 2.1 is the PostGIS driver: it connects to PostgreSQL database in order to obtain and/or update stored vector map layers. 


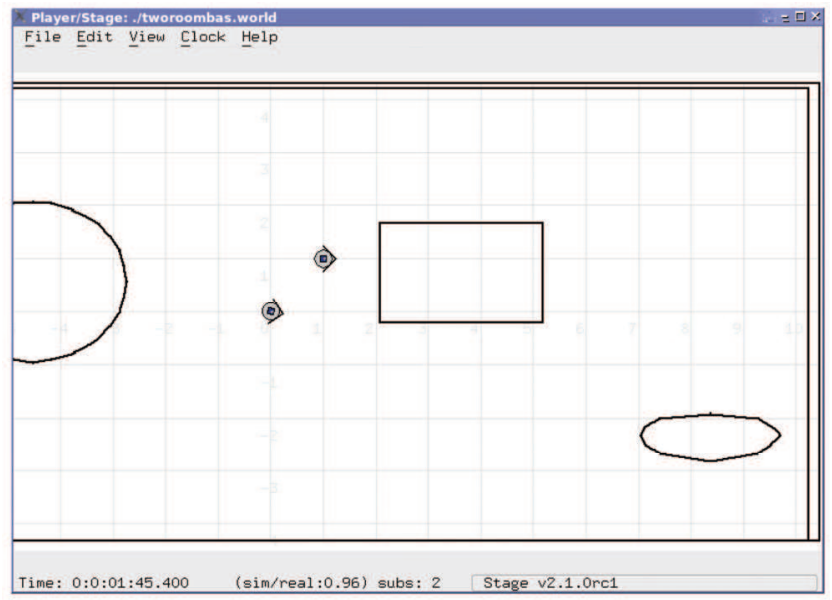

Figure 3. Stage simulator in use - two iRobot Roomba robots inside of simulated world

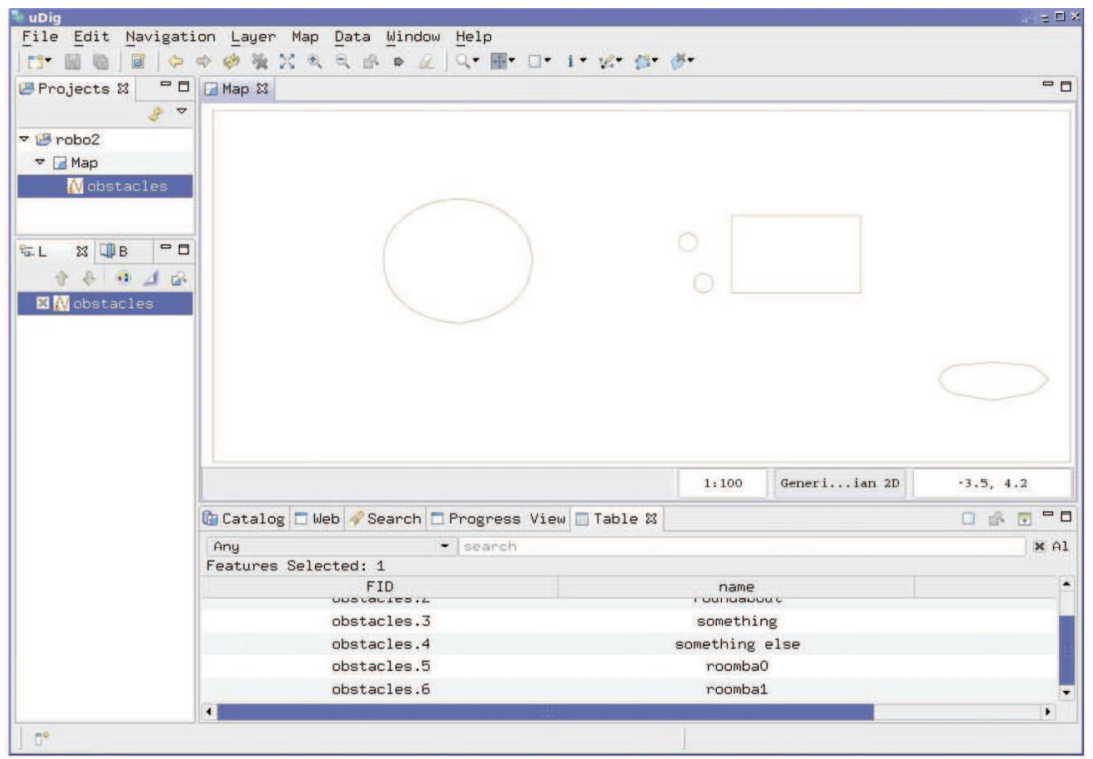

Figure 4. uDig application in use - modification of obstacles layer

PostGIS itself is an extension to the PostgreSQL object-relational database system which allows GIS (Geographies Information Systems) objects to be stored in the database [64]. It also offers new SQL functions for spatial reasoning. Maps which to be stored in SQL database can be created and edited by graphical tools like uDig or by $\mathrm{C} / \mathrm{C}++$ programs written using GEOS library of GIS functions. PostGIS, uDig and GEOS library are projects maintained by Refractions Research. 
A map can have many named layers, for each layer a table in SQL database is created. We can assume that layer named obstacles is full of objects that a robot cannot walk through. Other layers can be created in which we can divide robots workspace in areas with assigned attribute which for example tells if the given area is occupied by an obstacle or not. During our experimentations, we have created a plug-in for Players bunch of drivers which constantly tracks changes of position of every robot and updates obstacles layer such as robots are remarked as any other obstacle. As a result, the map stored in SQL database is kept always up to date. This feature is also useful in multi-agent environments: at any time a robot controller can send a query to SQL database server regarding every other robot position.

A roboticist can write a robot controller using Player client-side API which obtains information about current situation through the vectormap interface. Additionally, to write such a program, PostgreSQL client-side API can be used in order to open direct connection to the database server on which our mereoge-ometry SQL functions are stored together with map database. These functions can be called using this connection, results are sent back to the calling program. This gives robot controller program ability to perform spatial reasoning based on rough mereology.

Using PostGIS SQL extensions we have created our mereogeometry SQL functions [44]. Rough mereological distance is defined as such:

CREATE FUNCTION meredist(objectl geometry, object2 geometry)

RETURNS DOUBLE PRECISION AS

$\$ \$$

SELECT min(degrees.degree) FROM

((SELECT

ST_Area(STJntersection(extent $(\$ 1)$, extent $(\$ 2)))$

/ ST_Area(extent $(\$ 1))$

AS degree)

UNION (SELECT

ST_Area(STJntersection(extent $(\$ 1)$, extent $(\$ 2))$ )

/ ST_Area(extent $(\$ 2))$

AS degree))

AS degrees;

\$\$ LANGUAGE SQL STABLE;

Having mereological distance function we can derive nearness predicate:

CREATE FUNCTION merenear(obj geometry, ol geometry, o2 geometry)

RETURNS BOOLEAN AS

\$\$

SELECT meredist $(\$ 1, \$ 2)>\operatorname{meredist}(\$ 3, \$ 2)$

\$\$ LANGUAGE SQL STABLE;

The equi-distance can be derived as such:

CREATE FUNCTION mereequ(obj geometry, ol geometry, o2 geometry)

RETURNS BOOLEAN AS

\$\$

SELECT (NOT merenear $(\$ 1, \$ 2, \$ 3)$ )

AND (NOT merenear $(\$ 1, \$ 3, \$ 2)$ );

\$\$ LANGUAGE SQL STABLE; 
Our implementation of the betweenness predicate makes use of a function that produces an object which is an extent of given two objects:

CREATE FUNCTION mereextent(objectl geometry, object2 geometry)

RETURNS geometry AS

\$\$

SELECT GeomFromWKB(AsBinary(extent(objects.geom))) FROM

((SELECT \$1 AS geom)

UNION (SELECT \$2 AS geom))

AS objects;

\$\$ LANGUAGE SQL STABLE;

The betweenness predicate is defined as such:

CREATE FUNCTION merebetb(obj geometry, ol geometry, o2 geometry)

RETURNS BOOLEAN AS

\$\$

SELECT

meredist $(\$ 1, \$ 2)=1$

OR meredist $(\$ 1, \$ 3)=1$

OR

(meredist $(\$ 1, \$ 2)>0$

AND meredist $(\$ 1, \$ 3)>0$

AND meredist(mereextent $(\$ 2, \$ 3)$, mereextent $($ mereextent $(\$ 1, \$ 2), \$ 3))=1)$;

\$\$ LANGUAGE SQL STABLE;

Using the betweenness predicate we can check if three objects form a pattern:

CREATE FUNCTION merepattern

(objectl geometry, object2 geometry, objects geometry)

RETURNS BOOLEAN AS

\$\$

SELECT merebetb $(\$ 3, \$ 2, \$ 1)$

OR merebetb $(\$ 1, \$ 3, \$ 2)$

OR merebetb $(\$ 2, \$ 1, \$ 3)$;

\$\$ LANGUAGE SQL STABLE;

Also having pattern predicate we can check if four objects form a line:

CREATE FUNCTION mereisline4

(objl geometry, obj2 geometry, obj3 geometry, obj4 geometry)

RETURNS BOOLEAN AS

\$\$

SELECT merepattern $(\$ 1, \$ 2, \$ 3)$ AND merepattern $(\$ 2, \$ 3, \$ 4)$;

\$\$ LANGUAGE SQL STABLE;

Those predicates can be used in global navigation task. We can create additional map layer for navigational marker objects. Whenever the target is set, a robot planner should form a path across navigational markers. The path itself can be a group of objects representing areas free of obstacles. This group of objects in the path from the robot to the target should form a mereological line. A robot should follow this path by going from one area centroid to 
another until the goal is reached. If the changes in the world are expected (e.g. in multi-robot environments) a planner should update the path within some interval.

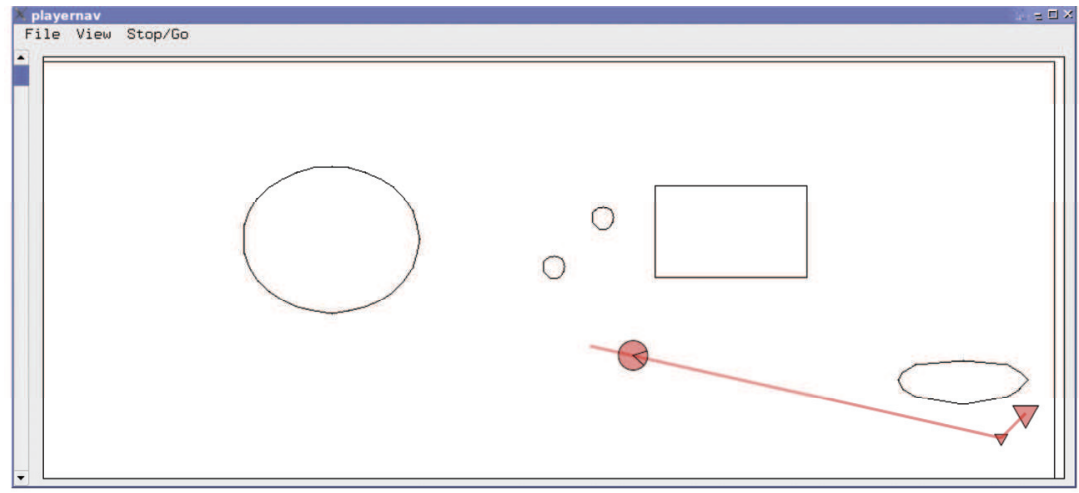

Figure 5. Playernav - a Player client-side application used to set a goal points for server-side planner driver

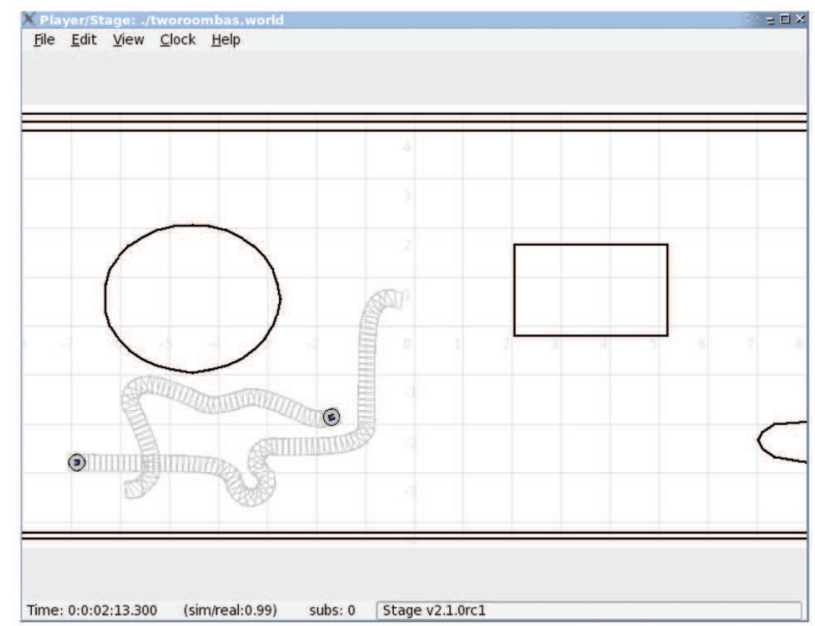

Figure 6. Show trails is a nice option in Stage which can be used to track robot trajectory. Here we can see two Roomba robots avoiding to hit obstacles and each other respectively. The robot controller program was using meredist function in order to choose free space area as a local target. This method of navigation suffers from local optima problem: a robot can start to spin around one place between obstacles

\section{References}

AISB-97: Spatial Reasoning in Mobile Robots and Animals, Proceedings AISB-97 Workshop, Manchester Univ., Manchester, 1997. [1]

J. Allen, Towards a general theory of action and time, Artificial Intelligence 23(20), 1984, pp. 123-154. [2] 
R. C. Arkin, Behavior-Based Robotics, MIT Press, Cambridge, MA, 1998. [3]

N.Asher, L. Vieu, Toward a geometry of commonsense: a semantics and a complete axiomatization of mereotopology, in: Proceedings IJCAI'95, Morgan KaufTman, San Mateo, CA, 1995, pp.846-852. [4]

N. Asher, M. Aurnague, M. Bras, P. Sablayrolles, L. Vieu, De 1'espace-temps dans 1'analyse du discours, Rapport interne IRIT/95-08-R, Institut de Recherche en Informatique, Univ. Paul Sabatier, Toulouse, 1995. [5]

M. Aurnague and L. Vieu, A theory of space-time for natural language semantics, in: K. Korta and J. M. Larrazabal, eds., Semantics and Pragmatics of Natural Language: Logical and Computational Aspects, ILCLI Series I, Univ. Pais Vasco, San Sebastian, 1995, pp. 69-126. [6]

W. Bandler, L. J. Kohout, Fuzzy power sets and fuzzy implication operators, Fuzzy Sets and Systems 4, 1980, 13-30. [7]

J. vanBenthem, The Logic of Time, Reidel, Dordrecht, 1983. [8]

T. Bittner, On ontology and epistemology of rough location, in: C. Freksa, D. M. Mark (eds.), Spatial Information Theory. Cognitive and Computational Foundations of Geographic Information Science, Lecture Notes in Computer Science vol. 1661, Springer Verlag, Berlin, 1999, pp. 433-448. [9]

G. Booch, Object-Oriented Analysis and Design with Applications, Addison-Wesley Publ., Menlo Park, 1994. [10]

R. Casati, B.Smith, A.C. Varzi, Ontological tools for geographic representation, in: N. Guarino (ed.), Formal Ontology in Information Systems, IOS Press, Amsterdam, 1998, pp. 77-85. [11]

B. L. Clarke, A calculus of individuals based on connection, Notre Dame Journal of Formal Logic 22(2), 1981, pp.204-218. [12]

R. Clay, Relation of Le'sniewski's Mereology to Boolean Algebra, The Journal of Symbolic Logic 39, 1974, pp. 638-648. [13]

A. G. Cohn, Calculi for qualitative spatial reasoning, in: J. Calmet, J. A. Campbell, J. Pfalzgraf (eds.), Artificial Intelligence and Symbolic Mathematical Computation, Lecture Notes in Computer Science vol. 1138, Springer Verlag, Berlin, 1996, pp. 124-143. [14]

A. G. Cohn, N. M. Gotts, Representing spatial vagueness: a mereological approach, in: Principles of Knowledge Representation and Reasoning: Proceedings of the 5th International Conference KR'96, Morgan Kaufmann, San Francisco, 1996, pp. 230-241. [15]

A. G. Cohn, A. C. Varzi, Connections relations in mereotopology, in: H. Prade (ed.), Proceedings ECAI'98. 13th European Conference on Artificial Intelligence, Wiley and Sons, Chichester, 1998, pp. 150-154. [16]

A. G. Cohn, N. M. Gotts, The "egg-yolk" representation of regions with indeterminate boundaries, in: P. Burrough, A. M. Frank (eds.), Proceedings GISDATA Specialist Meeting on Spatial Objects with Undetermined Boundaries, Fr. Taylor, 1996, pp.171-187. [17]

Collected Works of Stanislaw Lesniewski, J. Srzednicki, S. J. Surma, D. Barnett, V. F. Rickey (eds.), Kluwer, Dordrecht, 1992. [18]

E. Čech, Topological Spaces, Academia, Praha, 1966. [19] 
Z. Cui, A. G. Cohn, D. A. Randell, Qualitative and topological relationships, in: Advances in Spatial Databases, Lecture Notes in Computer Science vol. 692, Springer Verlag, Berlin, 1993, pp. 296-315. [20]

M. Dorigo, M. Colombetti, Robot Shaping. An Experiment in Behavior Engineering, MIT Press, Cambridge, MA, 1998. [21]

M. J. Egenhofer, R. G. Golledge (eds.), Spatial and Temporal Reasoning in Geographic Information Systems, Oxford U. Press, Oxford, 1997. [22]

C. Eschenbach, A predication calculus for qualitative spatial representations, in: C. Freksa, D. M. Mark (eds.), Spatial Information Theory. Cognitive and Computational Foundations of Geographic Information Science, Lecture Notes in Computer Science vol. 1661, Springer Verlag, Berlin, 1999, pp. 157-172. [23]

A. U. Frank, I. Campari (eds.), Spatial Information Theory: A Theoretical Basis for GIS, Lecture Notes in Computer Science, vol. 716, Springer Verlag, Berlin, 1993. [24]

A. U. Frank, W. Kuhn (eds.), Spatial Information Theory: A Theoretical Basis for GIS, Lecture Notes in Computer Science, vol. 988, Springer Verlag, Berlin, 1995. [25]

C. Freksa, D. M. Mark (eds.), Spatial Information Theory. Cognitive and Computational Foundations of Geographic Information Science. Proceedings COSIT'99, Lecture Notes in Computer Science vol. 1661, Springer Verlag, Berlin, 1999. [26]

C. Freksa, C. Habel, Repraesentation und Verarbeitung raeumlichen Wis-sens, InformatikFachberichte, Springer Verlag, Berlin, 1990. [27]

A. Galton The mereotopology of discrete space, in: C. Freksa, D. M. Mark (eds.), Spatial Information Theory. Cognitive and Computational Foundations of Geographic Information Science, Lecture Notes in Computer Science vol. 1661, Springer Verlag, Berlin, 1999, pp. 250-266. [28]

J. Glasgow, A formalism for model-based spatial planning, in: A. U. Frank, W. kuhn (eds.), Spatial Information theory - A Theoretical Basis for GIS, Lecture Notes in Computer Science vol. 988, Springer Verlag, Berlin, 1995, pp. 501-518. [29]

N. M. Gotts, A. G. Cohn, A mereological approach to representing spatial vagueness,in: Working papers, the Ninth International Workshop on Qualitative Reasoning, QR'95, 1995. [30]

N. M. Gotts, J. M. Gooday, A. G. Cohn, A connection based approach to commonsense topological description and reasoning, The Monist 79(1), 1996, pp. 51-75. [31]

N. Guarino, The ontological level, in: R. Casati, B. Smith, G. White (eds.), Philosophy and the Cognitive Sciences, Hoelder-Pichler-Tempsky, Vienna, 1994. [32]

S. C. Hirtle, A. U. Frank (eds.), Spatial Information Theory: A Theoretical Basis for GIS, Lecture Notes in Computer Science vol. 1329, Springer Verlag, Berlin, 1997. [33]

M. Inuiguchi, T. Tanino, Level cut conditioning approach to the necessity measure specification, in: Ning Zhong, A. Skowron, S. Ohsuga (eds.), New Directions in Rough Sets, Data Mining and Granular-Soft Computing, Lecture Notes in Artificial Intelligence vol. 1711, Springer Verlag, Berlin, 1999, pp. 193-202. [34]

B. Iwanus, On Lesniewski's Elementary Ontology, Studia Logica 31, 1973, pp. 73-119. [35]

T. Kotarbinski, Elements of the Theory of Knowledge, Formal Logic and Methodology of Science, Polish Sci. Publ., Warsaw, 1966. [36]

D. Kortenkamp, R. P. Bonasso, R. Murphy (eds.), Artificial Intelligence and Mobile Robots, AAAI Press/MIT Press, Menlo Park, CA, 1998. [37] 
R. Kruse, J. Gebhardt, F. Klawonn, Foundations of Fuzzy Systems, John Wiley \& Sons, Chichester, 1984. [38]

B. Kuipers, Qualitative Reasoning: Modeling and Simulation with Incomplete Knowledge, MIT Press, Cambridge MA, 1994. [39]

B. J. Kuipers, Y. T. Byun, A qualitative approach to robot exploration and map learning, in: Proceedings of the IEEE Workshop on Spatial Reasoning and Multi-Sensor Fusion, Morgan Kaufmann, San Mateo CA, 1987, pp. 390-404. [40]

B. J. Kuipers, T. Levitt, Navigation and mapping in large-scale space, AI Magazine 9(20), 1988, pp. 25-43. [41]

L. Kulik, A. Klippel, Reasoning about cardinal directions using grids as qualitative geographic coordinates, in: C. Freksa, D. M. Mark (eds.), Spatial Information Theory. Cognitive and Computational Foundations of Geographic Information Science, Lecture Notes in Computer Science vol.1661, Springer Verlag, Berlin, 1999, pp. 205-220. [42]

C. Kuratowski, Topology I, II, Academic Press and Polish Scientific Publishers, New YorkWarsaw, 1966. [43]

H. Ladanyi, SQL Unleashed, Sams Publishing, 1997. [44]

T. De Laguna, Point, line, surface as sets of solids, J. Philosophy 19, 1922, pp. 449-461. [45]

H. Leonard, N. Goodman, The calculus of individuals and its uses, The Journal of Symbolic Logic 5, 1940, pp. 45-55. [46]

Cz. Lejewski, On Lesniewski's Ontology, Ratio 1(2), 1958, pp. 150-176. [47]

Cz. Lejewski, A contribution to lesniewski's mereology, Yearbook for 1954-55 of the Polish Society of Arts and Sciences Abroad, V, 1954-55, pp. 43-50. [48]

St. Lesniewski, Grundziige eines neuen Systems der Grundlagen der Math-ematik, Fundamenta Mathematicae 24, 192 , pp. 242-251. [49]

St. Lesniewski, Uber die Grundlegen der Ontologie, C.R. Soc. Sci. Lettr. Varsovie III, 1930, pp.111-132. [50]

St. Lesniewski, On the Foundations of Mathematics, (in Polish), Przeglad Filozoficzny: 30,1927, pp. $164-206 ; 31,1928$, pp. 261-291; 32, 1929, pp. 60-101; 33, 1930, pp. 77-105; 34, 1931, pp. 142-170. [51]

St. Lesniewski On the foundations of mathematics, Topoi 2, 1982, pp. 7-52 (an abridged version of the preceding position). [52]

S. Marcus, Tolerance rough sets, Cech topologies, learning processes, Bull. Polish Acad. Ser. Sci. Tech 42(3), 1994, pp. 471-487. [53]

D. M. Mark, M. J. Egenhofer, K. Hornsby, Formal models of commonsense geographic worlds, NCGIA Tech. Report 97-2, Buffalo, 1997. [54]

C. Masolo, L. Vieu, Atomicity vs. infinite divisibility of space, in: C. Freksa, D. M. Mark (eds.), Spatial Information Theory. Cognitive and Computational Foundations of Geographic Information Science, Lecture Notes in Computer Science vol. 1661, Springer Verlag, Berlin, 1999, pp. 235-250. [55]

http: /www.c s. albany. edu/ amit[56]

P. Osmialowski, Player and Stage at PJIIT Robotics Laboratory, Journal of Automation, Mobile Robotics and Intelligent Systems, 2, 2007, pp. 21-28. [57]

L.Polkowski, On connection synthesis via rough mereology, Fundamenta Informaticae 46, 2001, pp. 83-96. [58] 
L. Polkowski, A rough set paradigm for unifying rough set theory and fuzzy set theory (a plenary lecture), Proceedings RSFDGrC'03), LNAI, vol. 2639, Springer-Verlag, Berlin, 2003, pp. 70-78. [59]

L. Polkowski, Toward rough set foundations. Mereological approach (a plenary lecture), Proceedings RSCTC04. LNAI, vol. 3066, Springer, Berlin, 2004, pp. 8-25. [60]

L. Polkowski and A. Skowron, Rough mereology: a new paradigm for approximate reasoning, International Journal of Approximate Reasoning 15(4), 1997, pp. 333-365. [61]

L. Polkowski, A. Skowron, Rough mereology in information systems with applications to qualitative spatial reasoning, Fundamenta Informaticae 43, 2000, pp. 291-320. [62]

L.Polkowski, A.Szmigielski, Computing with words via rough mereology in mobile robot navigation, Proceedings 2003 IEEE/RSJ Int. Conf. Intell. Robots and Systems IROS 2003, Las Vegas NV, 2003, pp. 3498-3503. [63]

P. Ramsey, PostGIS Manual, in: postgis.pdf file downloaded from Refractions Research home page. [64]

H. Reichenbach, The Philosophy of Space and Time (repr.), Dover, New York, 1957. [65]

R. Sikorski, Boolean Algebras, Springer Verlag, Berlin, 1960. [66]

P. Simons, Parts - A Study in Ontology, Clarendon, Oxford, 1987. [67]

A. Skowron and L. Polkowski, Rough mereological foundations for design, analysis, synthesis and control in distributed systems, Information Sciences. An Intern. J., 104, 1998, pp. 129-156. [68]

J. Slupecki, S. Lesniewski's Calculus of Names, Studia Logica 3, 1955, pp. 7-72. [69]

B. Smith, Logic and formal ontology, in: J. N. Mohanty, W. McKenna (eds.), Husserl's Phenomenology: A Textbook, Lanham: University Press of America, 1989, pp. 29-67. [70]

B. Smith, Agglomerations, in: C. Freksa, D. M. Mark (eds.), Spatial Information Theory. Cognitive and Computational Foundations of Geographic Information Science, Lecture Notes in Computer Science vol. 1661, Springer Verlag, Berlin, 1999, pp. 267282. [71]

B. Smith, Boundaries: an essay in mereotopology, in: L. Hahn (ed.), The Philosophy of Roderick Chisholm, Library of Living Philosophers, La Salle: Open Court, 1997, pp. 534-561. [72]

B. Sobociriski, Studies in Lesniewski's Mereology, Yearbook for 1954-55 of the Polish Society of Art and Sciences Abroad, 5 (1954), pp. 34-43. [73]

B. Sobocinski, L'analyse de 1'antinomie Russellienne par Lesniewski, Methodos, I, 1949, pp. 94-107, 220-228, 308-316; II, 1950, pp. 237-257. [74]

http:/agora.leeds.ac.uk/spacenet/spacenet.html[75]

J. G. Stell, Granulation for graphs, in: C. Freksa, D. M. Mark (eds.), Spatial Information Theory. Cognitive and Computational Foundations of Geographic Information Science, Lecture Notes in Computer Science vol. 1661, Springer Verlag, Berlin, 1999, pp. 416432. [76]

A. Szmigielski, A description based on rough mereology of the workspace of a mobile robot by means of a system of ultrasound sensors (in Polish), PhD Dissertation, Dept. Electronics and Computer Techniques, Warsaw University of Technology, 2003, L.Polkowski, supervisor. [77] 
A. Tarski, Les fondements de la geometrie des corps, in: Ksi§ga Pamiatkowa I Polskiego Zjazdu Matematycznego (Memorial Book of the 1st Polish Mathematical Congress), a supplement to Annales de la Societe Polonaise de Mathematique, Cracow, 1929, pp. 29-33. [78]

A. Tarski, Appendix E, in: J. H. Woodger, The Axiomatic Method in Biology, Cambridge Univ. Press, Cambridge, 1937. [79]

A. Tarski, What is elementary geometry?, in: L. Henkin, P. Suppes, A. Tarski (eds.), The Axiomatic Method with Special Reference to Geometry and Physics, Studies in Logic and Foundations of Mathematics, North-Holland, Amsterdam, 1959, pp. 16-29. [80]

A. Tarski, Zur Grundlegung der Boolesche Algebra I, Fundamenta Mathematicae 24, 1935, pp.177-198. [81]

T. Topaloglou, First-order theories of approximate space, in Working Notes of the AAAI Workshop on Spatial and Temporal Reasoning, Seattle, 1994, pp. 47-53. [82]

http:/www.cs.utexas.edu/users/qr/[83]

http:/www.cs.utexas.edu/users/qr/robotics/argus[84]

A. N. Whitehead, Process and Reality. An Essay in Cosmology, Macmillan, New York, 1929 (corr. ed. : D. R. Griffin, D. W. Sherbourne (eds.), 1978). [85]

M. F. Worboys, Imprecision in finite resolution spatial data, Geoinformatica 2, 1998, pp. 257279. [86] 


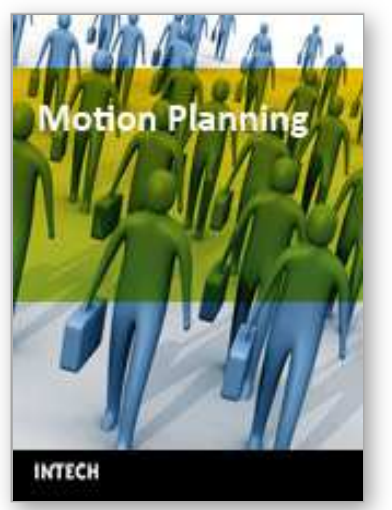

\author{
Motion Planning \\ Edited by Xing-Jian Jing
}

ISBN 978-953-7619-01-5

Hard cover, 598 pages

Publisher InTech

Published online 01, June, 2008

Published in print edition June, 2008

In this book, new results or developments from different research backgrounds and application fields are put together to provide a wide and useful viewpoint on these headed research problems mentioned above, focused on the motion planning problem of mobile ro-bots. These results cover a large range of the problems that are frequently encountered in the motion planning of mobile robots both in theoretical methods and practical applications including obstacle avoidance methods, navigation and localization techniques, environmental modelling or map building methods, and vision signal processing etc. Different methods such as potential fields, reactive behaviours, neural-fuzzy based methods, motion control methods and so on are studied. Through this book and its references, the reader will definitely be able to get a thorough overview on the current research results for this specific topic in robotics. The book is intended for the readers who are interested and active in the field of robotics and especially for those who want to study and develop their own methods in motion/path planning or control for an intelligent robotic system.

\title{
How to reference
}

In order to correctly reference this scholarly work, feel free to copy and paste the following:

Lech Polkowski and Pawel Osmialowski (2008). Spatial Reasoning with Applications to Mobile Robotics, Motion Planning, Xing-Jian Jing (Ed.), ISBN: 978-953-7619-01-5, InTech, Available from:

http://www.intechopen.com/books/motion_planning/spatial_reasoning_with_applications_to_mobile_robotics

\section{INTECH}

open science | open minds

\section{InTech Europe}

University Campus STeP Ri

Slavka Krautzeka 83/A

51000 Rijeka, Croatia

Phone: +385 (51) 770447

Fax: +385 (51) 686166

www.intechopen.com

\section{InTech China}

Unit 405, Office Block, Hotel Equatorial Shanghai

No.65, Yan An Road (West), Shanghai, 200040, China

中国上海市延安西路65号上海国际贵都大饭店办公楼405单元

Phone: +86-21-62489820

Fax: +86-21-62489821 
(C) 2008 The Author(s). Licensee IntechOpen. This chapter is distributed under the terms of the Creative Commons Attribution-NonCommercialShareAlike-3.0 License, which permits use, distribution and reproduction for non-commercial purposes, provided the original is properly cited and derivative works building on this content are distributed under the same license. 